\title{
Self-Assembly Engineering
} Nanodrugs Composed of Paclitaxel and Curcumin for the Combined Treatment of Triple Negative Breast Cancer

\author{
Shuting Zuo ${ }^{1}$, Zhenyu Wang ${ }^{1}$, Xianquan $\mathrm{An}^{2}$, Jing Wang ${ }^{1}$, Xiao Zheng ${ }^{3}$, Dan Shao ${ }^{3 *}$ and \\ Yan Zhang ${ }^{1 *}$
}

${ }^{1}$ Department of Breast Surgery, The Second Hospital of Jilin University, Changchun, China, ${ }^{2}$ Department of Anesthesiology, The Second Hospital of Jilin University, Changchun, China, ${ }^{3}$ School of Biomedical Sciences and Engineering, South China University of Technology, Guangzhou, China

\section{OPEN ACCESS}

Edited by:

Jinbing Xie,

Southeast University, China

Reviewed by:

Jinfeng Liao,

Sichuan University, China

Qianyu Zhang,

Chongaing University, China

${ }^{*}$ Correspondence:

Yan Zhang

zhangy01@jlu.edu.cn

Dan Shao

stanauagate@outlook.com

Specialty section:

This article was submitted to

Nanobiotechnology,

a section of the journal

Frontiers in Bioengineering and

Biotechnology

Received: 26 July 2021 Accepted: 11 August 2021 Published: 24 August 2021

Citation:

Zuo S, Wang Z, An X, Wang J, Zheng $X$, Shao $D$ and Zhang $Y$ (2021) Self-Assembly Engineering Nanodrugs Composed of Paclitaxel and Curcumin for the Combined Treatment of Triple

Negative Breast Cancer.

Front. Bioeng. Biotechnol. 9:747637.

doi: 10.3389/fbioe.2021.747637
The clinical outcomes of triple-negative breast cancer (TNBC) chemotherapy are unsatisfactory. Water solubility and biosafety of chemo drugs are also major barriers for achieving satisfactory treatment effect. In this study, we have reported a combinational strategy by self-assembly engineering nanodrugs PC NDs, which were composed of paclitaxel (PTX) and curcumin (Cur), for effective and safe TNBC chemotherapy. PC NDs were prepared through reprecipitation method without using any additional carriers. The PC NDs were preferentially taken up by TNBC cells and we also observed $\mathrm{pH}$-related drug release. Compared with free PTX and simple PTX/Cur mixture, PC NDs have shown higher therapeutic efficiency and better prognosis while the metastasis rate was significantly lower than that of either PTX or PTX/Cur mix group. Therefore, the self-assembly engineered PC NDs might be a promising nanodrugs for efficient and safe TNBC chemotherapy.

Keywords: triple-negative breast cancer, paclitaxel, curcumin, self-assembly, nanodrug

\section{INTRODUCTION}

Breast cancer is the most common cancer type among women worldwide and threatens their health seriously (McDonald et al., 2016; Yeo and Guan, 2017; Fahad Ullah, 2019). Many chemotherapeutic, hormone-based, and combination drug regimens have been used to treat breast cancer, although patients with advanced aggressive disease (especially triple-negative breast cancer, TNBC) still have poor survival outcomes (Bergin and Loi, 2019). In this context, TNBC is characterized by non-expression of protein receptors, including progesterone receptor, estrogen receptor, and human epidermal growth receptor 2 . Relative to other subtypes, TNBC is more aggressive, has a poorer prognosis and higher rates of visceral and central nervous system metastases, with no currently approved targeted therapies (Kumar and Aggarwal, 2016; Akram et al., 2017). Chemotherapy, including neoadjuvant chemotherapy, remains the only standard treatment option for TNBC, although patients have a poor response because of rapidly acquired drug resistance and distant metastasis, and also inevitably experience adverse effects (Lyons, 2019; Yin et al., 2020). Thus, there is a need for combination therapies that can improve the efficacy of current chemotherapeutic strategies for TNBC.

Paclitaxel (PTX) is a first-line chemotherapy drug that is used to treat TNBC by preventing microtubule depolymerization and arresting mitosis at G2/M stages of cell cycle. However, paclitaxel 
has poor solubility, unavoidable toxicity, and tumors can develop resistance (Bielopolski et al., 2017; Abu Samaan et al., 2019). There is growing evidence that combining PTX with other chemotherapeutic agents (e.g., small molecule inhibitors or natural products) could enhance the anti-tumor effect (Schmid et al., 2018; Kang and Syed, 2020; Mittendorf et al., 2020). Curcumin (Cur) is a hydrophobic polyphenol derived from Curcuma longa (the spice turmeric). It exhibits anti-bacterial, anti-inflammatory, and anti-cancer properties. Furthermore, Cur has been proved to have excellent safety and widespread availability with low cost. Previous studies have indicated that Cur can downregulate the NF-kB and PI3K/Akt signaling pathways in cancer cells, which can inhibit cell growth, induce apoptosis, and increase drug sensitization (Hamzehzadeh et al., 2018; Subramaniam et al., 2018; Chen et al., 2019; Ghasemi et al., 2019; Maiti et al., 2019; Zusso et al., 2019; Borges et al., 2020; Keyvani-Ghamsari et al., 2020). In addition, the combination of PTX and Cur provided synergistic anti-cancer effects and eliminated cancer stem cells in TNBC (Baek and Cho, 2017; Calaf et al., 2018; Saghatelyan et al., 2020). However, similar to PTX, Cur has poor solubility and low bioavailability, which has limited its clinical application. Therefore, it would be useful to develop an efficient and safe system that could simultaneously deliver PTX and Cur to treat TNBC.

Significant work has been dedicated to developing drug delivery system that can concurrently deliver PTX and Cur to the tumor site, which might provide improved therapeutic activity and safety. However, most reported carriers have limited loading capacity and there are also concerns regarding their possible toxicity and biodegradation (Hiremath et al., 2019; Li et al., 2019; Zhao et al., 2019; Shao et al., 2020; Xiong et al., 2020; Hu et al., 2021; Liao et al., 2021; Zhang et al., 2021). Carrierfree drug delivery systems are recently developed alternatives that do not rely on inert carriers and thus avoid potential toxicity. Common strategies for preparing carrier-free nanodrugs include nanoprecipitation, thin-film hydration, template-assisted nanoprecipitation, supercritical fluid techniques, spray drying, and wet media milling (Zhang et al., 2018; Zheng et al., 2018; Sun et al., 2019; Zhou et al., 2019). Relative to free drugs, carrier-free nanodrugs have prolonged blood circulation times, better cellular penetration, and greater tumor accumulation (Yang et al., 2019), which has generated interest regarding their clinical applications. Therefore, we developed a carrier-free nanodrug that is composed of PTX and Cur (PC NDs) using a one-pot self-assembly nanoprecipitation method. Physicochemical, optical and drug release properties of PC NDs were characterized, and we then evaluated their effects against TNBC cells in vitro and in vivo. The results suggest that PC NDs may be a highly effective and safe option for treating TNBC.

\section{MATERIALS AND METHODS}

\section{Chemicals and Reagents}

Cur (purity: >94\%) and sulforhodamine B (SRB) were obtained from Sigma-Aldrich (St. Louis, MO, United States). PTX was purchased from Solarbio Science and Technology Co., Ltd.
(Beijing, China). Dulbecco's Modified Eagle Medium (DMEM), fetal bovine serum (FBS), trypsin and penicillinstreptomycin $(10,000 \mathrm{U} / \mathrm{ml})$ were obtained from GIBCO (Carlsbad, CA, United States). Matrigel was purchased from Corning Inc. (Billerica, MA, United States). Hoechst 33,258 and Lysotracker Red were purchased from Thermo Fisher Scientific (Waltham, MA, United States). Assay kits for determing alanine aminotransferase (ALT), aspartate aminotransferase (AST), alkaline phosphatase (ALP), blood urea nitrogen (BUN), and creatinine (CRE) were obtained from Nanjing Jiancheng Bioengineering Institute (Nanjing, Jiangsu, China). All reagents were directly used without any further purification.

\section{Preparation and Characterization of PC NDs}

The PC NDs were prepared using a reprecipitation method. First, Cur and PTX were dissolved in ethyl alcohol to provide solutions with concentrations of $2 \mathrm{mg} / \mathrm{ml}$. Next, $0.4 \mathrm{ml}$ of the PTX solution and $0.1 \mathrm{ml}$ of the Cur solution were quickly added to $4.5 \mathrm{ml}$ of deionized water, vortexed for $1 \mathrm{~min}$ and allowed to stand for 15 min to produce PC NDs. Finally, PC NDs were purified via ultrafiltration and collected via lyophilization, which provided a 4:1 weight ratio (PTX to Cur) after quantified by UV-vis method.

The morphology of PC NDs was inspected by a transmission electron microscope (JEOL, Ltd., Japan) and a scanning electron microscope (FESEM, S4800, Hitachi Co. Ltd., Tokyo, Japan). Fluorescence spectroscopy was performed using a Shimadzu RF5301 PC spectrophotometer. UV-vis absorption spectra were obtained using a Shimadzu 3100 UV-vis spectrophotometer. Fourier transform infrared (FTIR) spectra were performed with a Nicolet AVATAR 360 FTIR instrument. X-ray powder diffraction (XRD) investigation was carried out on a Rigaku X-ray diffractometer using $\mathrm{Cu}$ Ka radiation. A Nano-ZS 90 Nanosizer (Malvern Instruments Ltd., Worcestershire, United Kingdom) was used to determine the size distribution and zeta potential of PC NDs.

\section{Drug Release}

Drug release behavior was evaluated by adding $5 \mathrm{mg}$ of the PC NDs to a dialysis bag $(5,000 \mathrm{Da})$, which was then placed in $50 \mathrm{ml}$ of phosphate-buffered saline solution (PBS, pH: 7.4 or 5.5) on a shaking table at $37^{\circ} \mathrm{C}$ for $48 \mathrm{~h}$. Supernatant was then collected and the amounts of PTX and Cur were analyzed via highperformance liquid chromatography.

\section{Cell Culture and Uptake}

A mouse breast cancer cell line (4T1), a human TNBC cell line (MDA-MB-231), and a non-neoplastic breast cell line (MCF-10A) were purchased from the American Type Culture Collection. All cells were cultured in DMEM with $10 \%$ FBS, $100 \mathrm{U} / \mathrm{ml}$ penicillin and $100 \mathrm{U} / \mathrm{ml}$ streptomycin in a humidified incubator with an atmosphere of $5 \% \mathrm{CO}_{2}$. Cellular uptake of PC NDs was evaluated after a 3-h incubation with cells, which were then washed with PBS and co-incubated with Lysotracker Red and Hoechst 33,258. Then cells were observed under an Olympus 
IX71 fluorescence microscope (Olympus Corporation, Tokyo, Japan). Quantification of cellular uptake was conducted through flow cytometry (FACS, Becton Dickinson Biosciences, Franklin Lakes, United States).

\section{Cytotoxicity}

Cells were first seeded into 96-well culture plates at the density of 5,000 cells per well and then cultured overnight for fully attaching. Free Cur and PTX were dissolved deionized water at a concentration of $1 \mathrm{mg} / \mathrm{ml}$, respectively. Next cells were treated with different final concentrations of free PTX, free Cur, PTX/Cur mixture (PTX/Cur mix) or PC NDs. After being treated for 24 or $48 \mathrm{~h}$, cells were subjected to standard $\mathrm{SRB}$ assay and absorbance at $540 \mathrm{~nm}$ was analyzed using a multifunctional microplate reader. $\mathrm{IC}_{50}$ values were calculated by GraphPad Prism software.

\section{In vivo Experiments}

All mice were treated in compliance with the Guide for the Care and Use of Laboratory Animals, and all procedures were approved by the Animal Care and Use Committee of Jilin University (China). $50 \mu \mathrm{L}$ of $4 \mathrm{~T} 1$ cells $\left(5 \times 10^{5}\right)$ were mixed with $50 \mu \mathrm{L}$ of matrigel and then orthotopically injected into the second mammary fat pad of female $\mathrm{BALB} / \mathrm{c}$ mice (six to eight weeks old). Mice were randomly divided into five groups $(\mathrm{n}=6)$ when tumor volume reached approximately $100 \mathrm{~mm} 3$, which were treated using saline, PTX $(10 \mathrm{mg} / \mathrm{kg}$, intraperitoneally), Cur (2.5 mg/kg, intraperitoneally), PTX/Cur mix (10 mg/kg of PTX and $2.5 \mathrm{mg} / \mathrm{kg}$ of Cur, intraperitoneally), or PC NDs $(10 \mathrm{mg} / \mathrm{kg}$, intravenously), respectively. All drug treatments were administered every 3 days, and measurements of tumor volume and body weight were performed at the same time. Tumor volume was calculated according to the following formula: volume $=0.5 \times$ (longest dimension) $\times$ (shortest dimension $)^{2}$.

After 21 days of treatment, all mice were sacrificed on day 22. Tumors were measured and weighed. Main organs (liver, spleen, kidneys, hearts, and lungs) were collected, fixed and stained using hematoxylin and eosin ( $\mathrm{H}$ and $\mathrm{E}$ ) before being photographed. Biosafety was evaluated based on changes in body weight, pathological changes in organs mentioned above and serum biochemistry indexes including ALT, AST, ALP, BUN, and CRE.

\section{Statistical Analysis}

All experiments were performed at least three times and results were exhibited as mean \pm standard deviation. Comparison between groups were calculated by Student's t-test (two groups) or Bonferroni's post hoc test (three groups or more). Data were analyzed on SPSS software. Differences were considered statistically significant when $p$-values were less than 0.05 .

\section{RESULTS AND DISCUSSION}

The PC NDs were created by quickly adding ethanol solution containing PTX and Cur into excessive volume of deionized water and vortexing the mixture for $1 \mathrm{~min}$ (Figure $\mathbf{1 A}$ and Supplementary Figure S1). During the nanoprecipitation process, PTX, and Cur molecules were precipitated to form nanoparticles via intermolecular interactions, such as hydrogen bonding, $\pi-\pi$ stacking and hydrophobic interactions (Li et al., 2018; Hupfer et al., 2021). The PC NDs had a spherical structure (diameter: $120-140 \mathrm{~nm}$ ) based on characterization via TEM and SEM (Figures 1B,C). Zeta potential measurements revealed that the PC NDs had a negative surface charge $(-14.6 \pm 0.513 \mathrm{mV})$, which was similar to that of raw Cur $(-22.2 \pm 3.96 \mathrm{mV})$ and suggested that the surface of the PC NDs was mostly composed of Cur with phenolic hydroxyl groups. The ultraviolet-visible light absorption and fluorescence spectra of free PTX, free Cur, free PTX/Cur mix, and PC NDs were analyzed. The PC NDs had the same absorption peaks as free PTX and Cur, albeit with variable peak heights (Figures 1D,E). Furthermore, PC NDs have exhibited weaker green fluorescence than free Cur when excited with 420-nm laser, although free Cur, free PTX/Cur, and PC NDs shared the same emission peak $(550 \mathrm{~nm}$, Figure 1D). We used FTIR spectra to evaluate whether the bioactive groups of PTX and Cur were preserved in the PC NDs (Figure 1F), which revealed that the PC NDs exhibited $\mathrm{N}-\mathrm{H}$ stretching vibration at $3,515 \mathrm{~cm}^{-1}$ and $\mathrm{C}=\mathrm{C}$ stretching vibration of the conjugate system in Cur and PTX at $1,513 \mathrm{~cm}^{-1}$, as well as disappearance of the strong $\mathrm{O}-\mathrm{H}$ stretching vibration at $3,511 \mathrm{~cm}^{-1}$. Based on these results, we conclude that the PC NDs contained most of the bioactive groups of PTX and Cur, with Cur potentially being "surrounded" by PTX to create the spherical structure of the PC NDs. In addition, we performed XRD measurements to determine whether the PC NDs formed drug eutectics or amorphous formations (Figure 1G), which revealed that the PC NDs had an XRD spectrogram that was similar to that of PTX crystal, with amorphous Cur structures. These findings suggest that the PC NDs were probably composed of crystalline formations containing PTX nanocrystals and Cur.

The release profiles of PTX and Cur from PC NDs were evaluated using a dialysis bag and $\mathrm{PBS}$ solution, with a $\mathrm{pH}$ value of 7.4 to mimic normal conditions in bodily fluids or a $\mathrm{pH}$ value of 5.5 to mimic the acidic tumor microenvironment, respectively. As shown in Figure 2A, PTX release showed a time-dependent behavior and reached approximately $33.3 \%$ over a 48 -h period at a $\mathrm{pH}$ of 7.4. However, when the $\mathrm{pH}$ was 5.5, PTX release increased substantially and reached $83.2 \%$ over a 48 -h period. Similar trends were observed in the acid- and time-dependent release of Cur (Figure 2B). Thus, the PC NDs might facilitate simultaneous and preferred release of PTX and Cur in the acidic tumor microenvironment. As shown in Supplementary Figure S2, PC NDs disintegrated quickly in acidic PBS, while the hydration radius has increased by about $25 \%$ during $72 \mathrm{~h}$ in neutral PBS. Therefore, PCNDs were used just after preparation. Fluorescence microscopy was subsequently used to evaluate cellular uptake of the two drugs. After incubation of 4T1 and MDA-MB-231 cells with PC NDs for $2 \mathrm{~h}$, we labelled the nuclei with Hoechst dye (blue) and lysosomes with LysoTracker RED DND (red). Green fluorescence (Cur) was observed in the cytoplasm and lysosomes (Figure 3B and Supplementary 
A

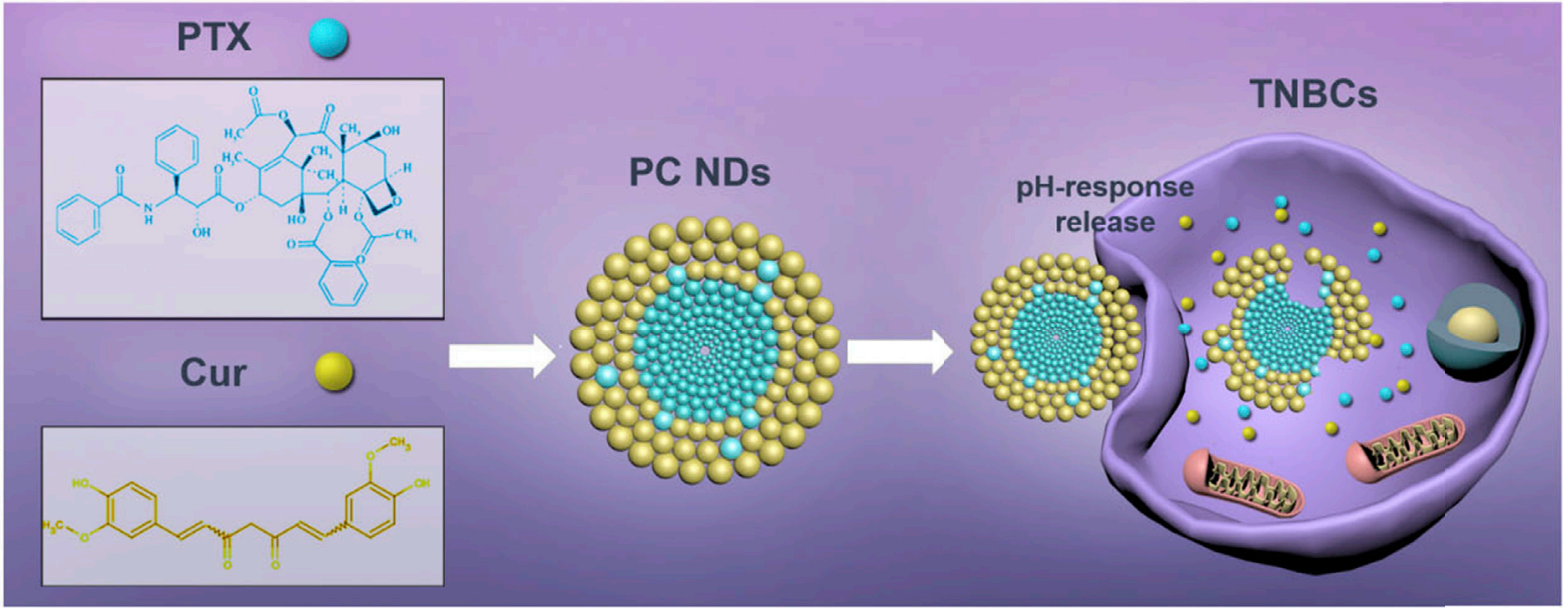

B

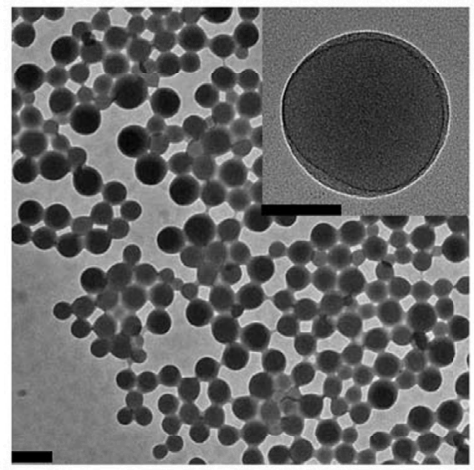

E

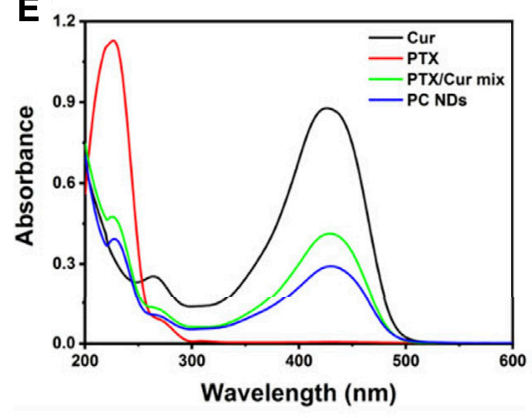

C

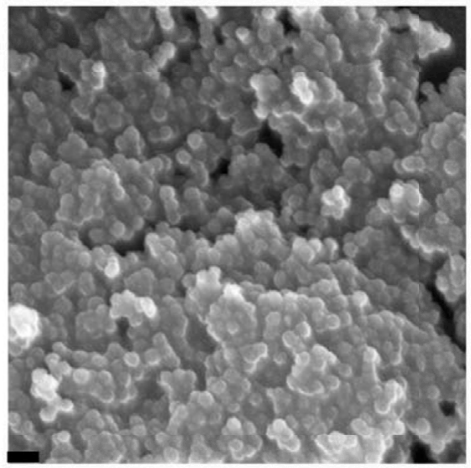

F

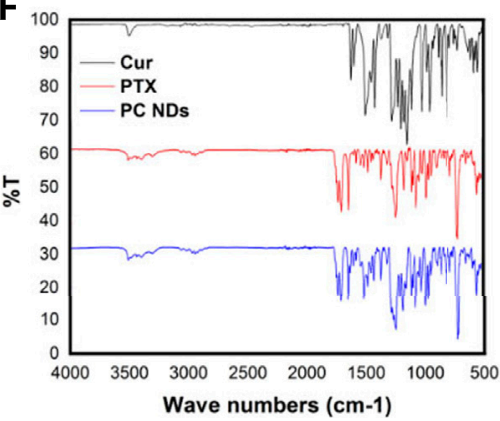

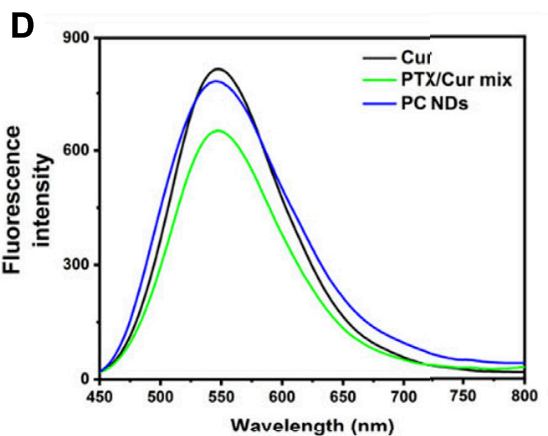

G

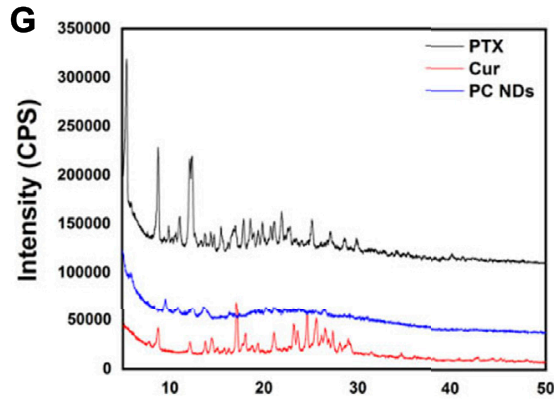

FIGURE 1 | Scheme of PC NDs preparation and its characterization. (A) Scheme of PC NDs preparation. PC NDs has been prepared using a reprecipitation method and shown pH-sensitive drug release behavior in tumor cells. (B) TEM images of PC NDs. Scale bars are 200 and $50 \mathrm{~nm}$ (inset), respectively. (C) SEM image of PC NDs. Scale bar is 200 nm. (D) Emission spectra of Cur, PTX/Cur mix, and PC NDs. (E) UV-vis absorption spectra of Cur, PTX, PTX/Cur mix, and PC NDs. (F) FTIR spectra of Cur, PTX, and PC NDs. (G) XRD spectra of Cur, PTX, and PC NDs.

Figure S3), and the overlap of the green and red fluorescence signals suggested that the PC NDs were accumulated in lysosomes. Furthermore, the intensity of the PC ND staining increased with incubation time in the TNBC cells (Figure 3A), which suggested a time-dependent drug release mechanism. As expected, the PC NDs had stronger fluorescence intensity (vs free Cur), which confirmed their higher rate of cellular uptake. However, relative to in the cancer cells, there was significantly less Cur uptake into normal breast cells (MCF-10A), which is likely related to the higher $\mathrm{pH}$ in normal cells. When considered together, these findings indicate that PC NDs can be taken up by TNBC cells and their contents released in a $\mathrm{pH}$-dependent manner.

SRB assay was used to determine the viability of $4 \mathrm{~T} 1, \mathrm{MDA}-$ MB-231, and MCF-10A cells after 24-48 h of treatment using various concentrations of free PTX, free PTX/Cur mix, and PC NDs. Relative to the control group, 4T1 cell viability of all PTX-containing groups has exhibited a dose- and timedependent decrease manner (Figures 4A,B), while the addition of Cur provided even greater decreases. Similar 

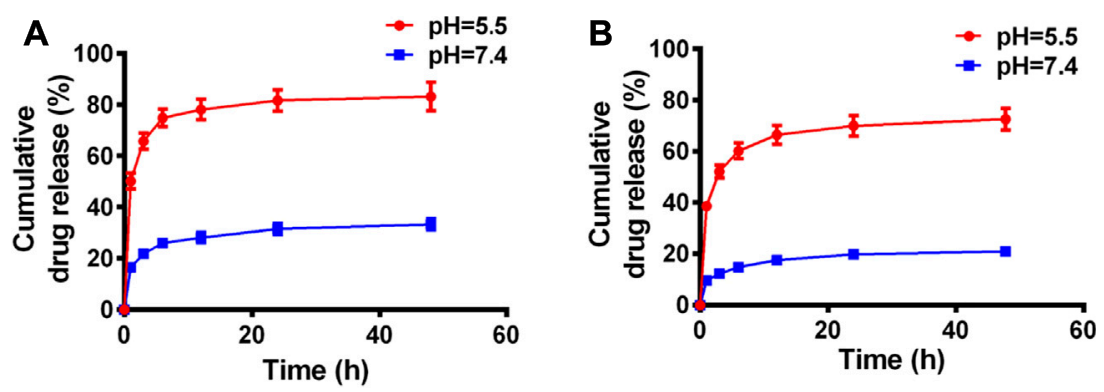

FIGURE 2 | Drug release profiles of PC NDs in PBS of different $\mathrm{pH}$ values during $48 \mathrm{~h}$ (A) PTX and (B) Cur release of PC NDs in neutral and acidic PBS.

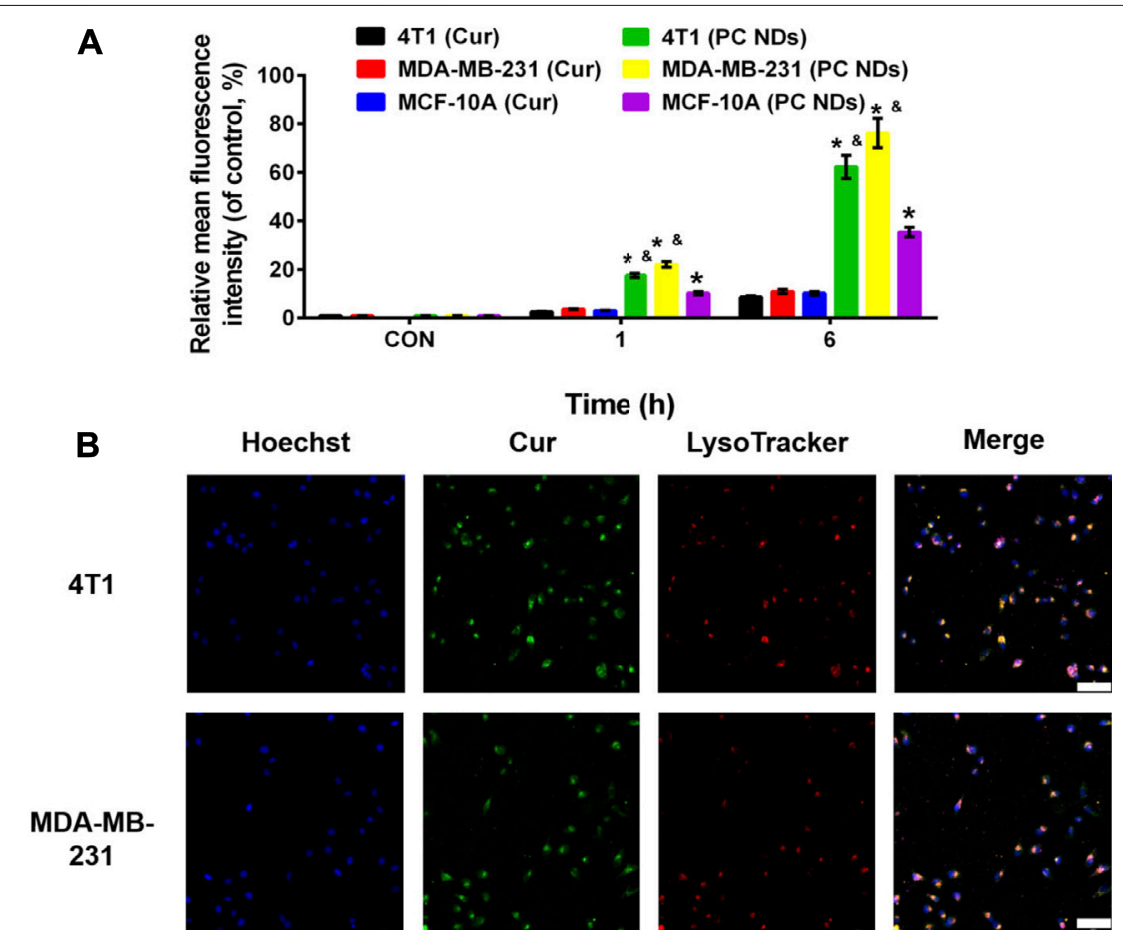

FIGURE 3 | Cellular uptake of PC NDs in 4T1, MDA-MB-231, and MCF-10A cells. (A) Quantitative analysis of the internalization of Cur and PC NDs in 4T1, MDAMB-231, and MCF-10A cells through FACS. Statistical significance: ${ }^{*} p<0.05$ vs control and $p<0.05$ vs Cur.(B) CLSM images of 4 T1 and MDA-MB-231 cells after incubation with PC NDs for $2 \mathrm{~h}$. Scale bars are $200 \mu \mathrm{m}$.

results were also observed in MDA-MB-231 cells (Figures 4C,D). At $48 \mathrm{~h}$, the $\mathrm{IC}_{50}$ values in $4 \mathrm{~T} 1$ cells were $16.52 \pm 0.16 \mu \mathrm{M}$ for free PTX, $4.05 \pm 0.13 \mu \mathrm{M}$ for PTX/Cur mix, and $3.87 \pm 0.14 \mu \mathrm{M}$ for PC NDs. Similarly, the IC50 values in MDA-MB-231 cells at $48 \mathrm{~h}$ were $7.34 \pm 0.19 \mu \mathrm{M}$ for free PTX, $2.79 \pm 0.10 \mu \mathrm{M}$ for PTX/Cur mix, and $2.58 \pm 0.11 \mu \mathrm{M}$ for PC NDs. Moreover, to our expect, cells incubated with Cur have shown little to no decease on cell viability due to its low concentration (Supplementary Figure S4). These results suggest that combining PTX and Cur provided a significantly greater decrease in TNBC cell viability, relative to PTX alone, which might be related to Cur-induced sensitization of the TNBC cells to PTX (Saha et al., 2012; Yoshida et al., 2017). The small differences between the PTX/Cur mix and PC NDs might be the result of long treatment time, which has given enough time for cells to uptake nearby drugs. It is also worth noting that the PC NDs had less effect on MCF-10A cells than free PTX (Figures 4E,F), which might be related to preferred $\mathrm{pH}$ related drug release in cancer cells (vs in normal cells). When considered together, these results suggest that the PC NDs provided a greater decrease in TNBC cell viability (vs PTX alone), as well as less toxicity in normal cells.

The BALB/c mice bearing 4T1 tumors were treated using saline (control group), free Cur, free PTX, free PTX/Cur mix, and PC NDs (Figure 5A). Relative to control group, decreased tumor growth and lower tumor weights were observed at the end of treatment using free PTX, free PTX/Cur mix, and PC NDs 


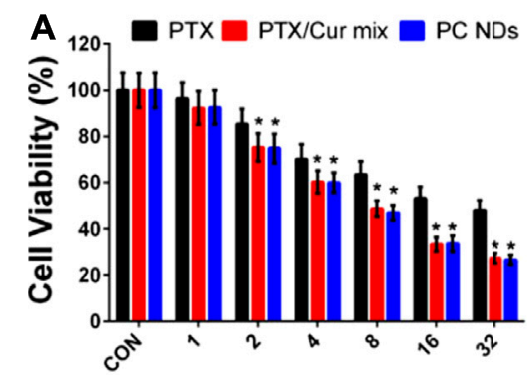

Concentration $(\mu \mathrm{M})$

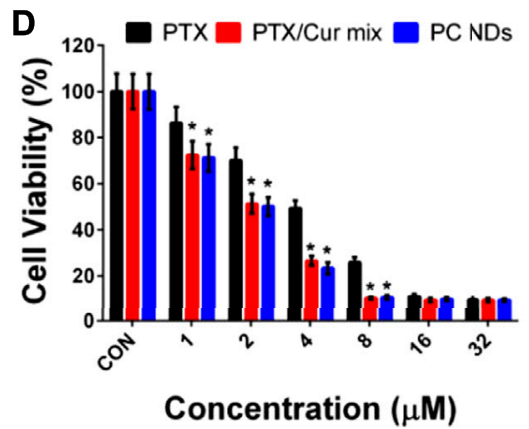

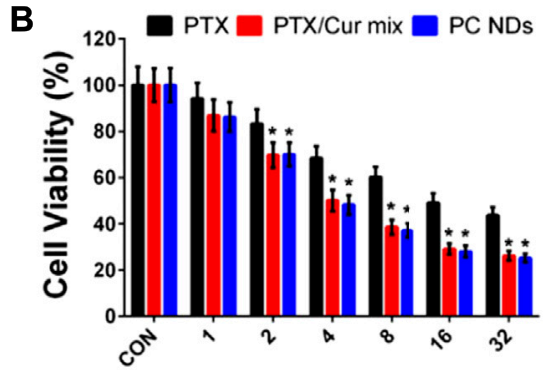

Concentration $(\mu \mathrm{M})$

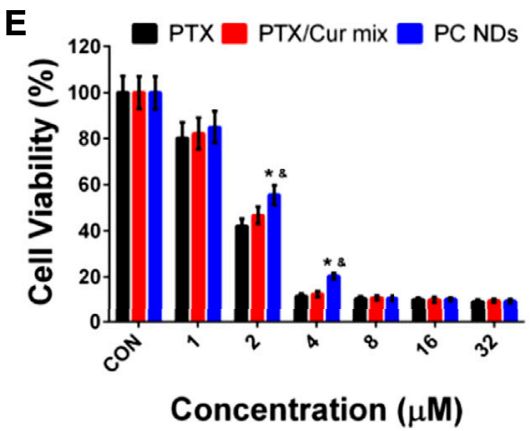

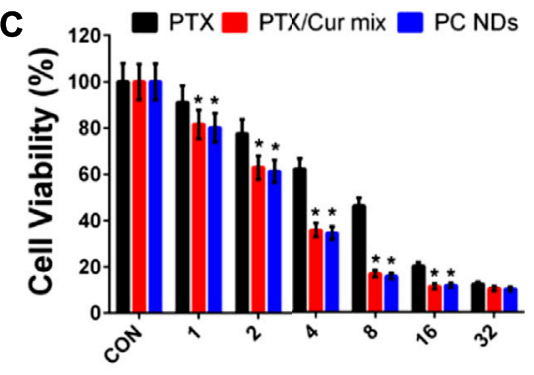

Concentration $(\mu \mathrm{M})$

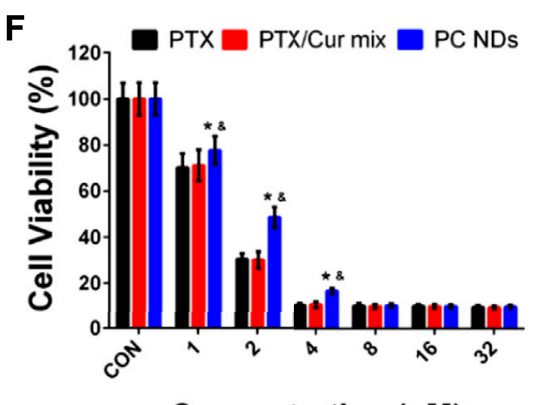

Concentration $(\mu \mathrm{M})$

FIGURE 4 | Cytotoxicity of PTX, PTX/Cur mix, and PC NDs in 4T1, MDA-MB-231 and MCF-10A cells after 24 and 48 h incubation. Cell viability of (A) 4 T1 cells, (C) MDA-MB-231 cells, and (E) MCD-10A cells after $24 \mathrm{~h}$ incubation. Cell viability of (B) $4 \mathrm{T1}$ cells, (D) MDA-MB-231 cells, and (F) MCD-10A cells after $48 \mathrm{~h}$ incubation. Statistical significance: ${ }^{*} p<0.05$ vs control and $p<0.05$ vs PTX.

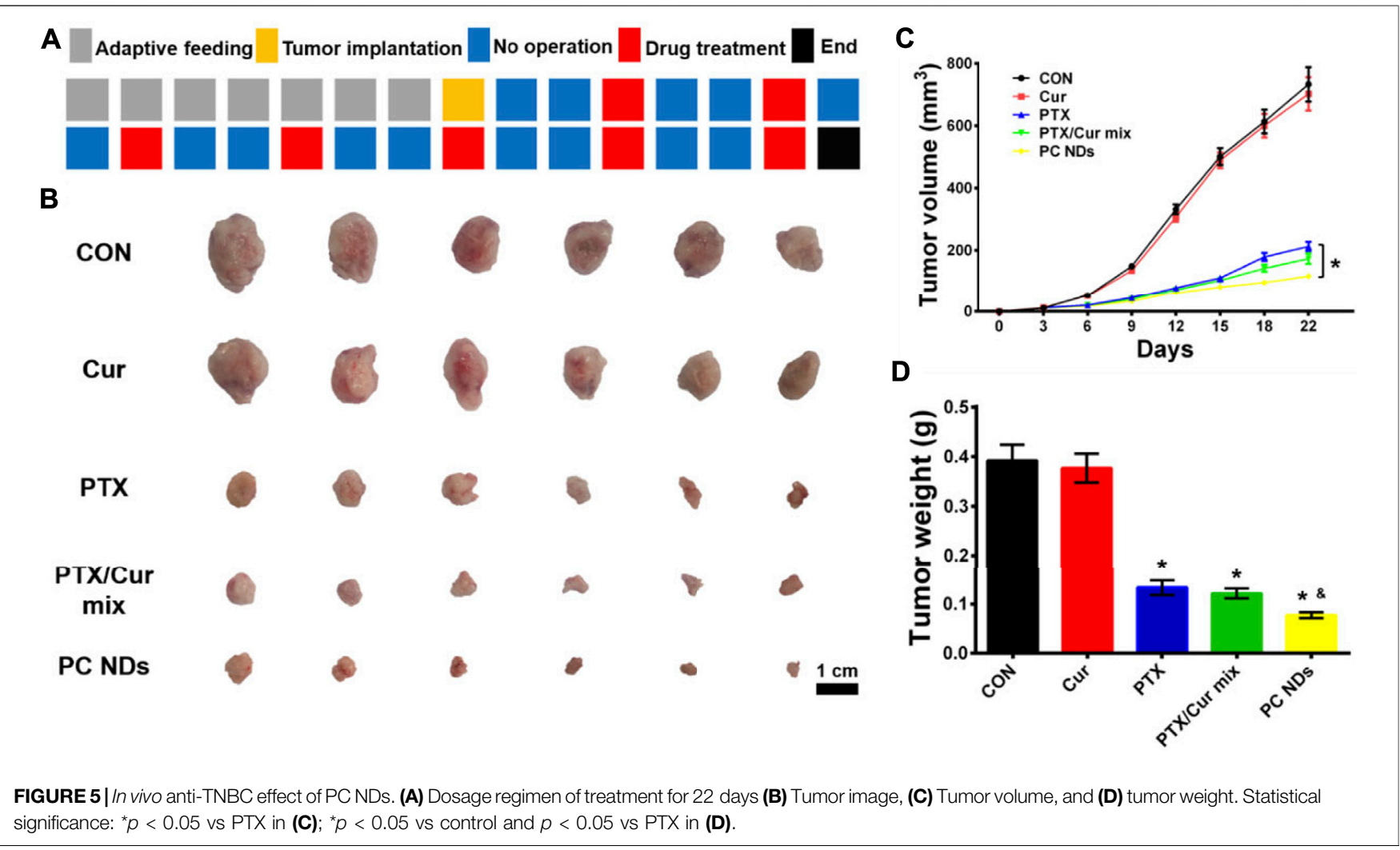




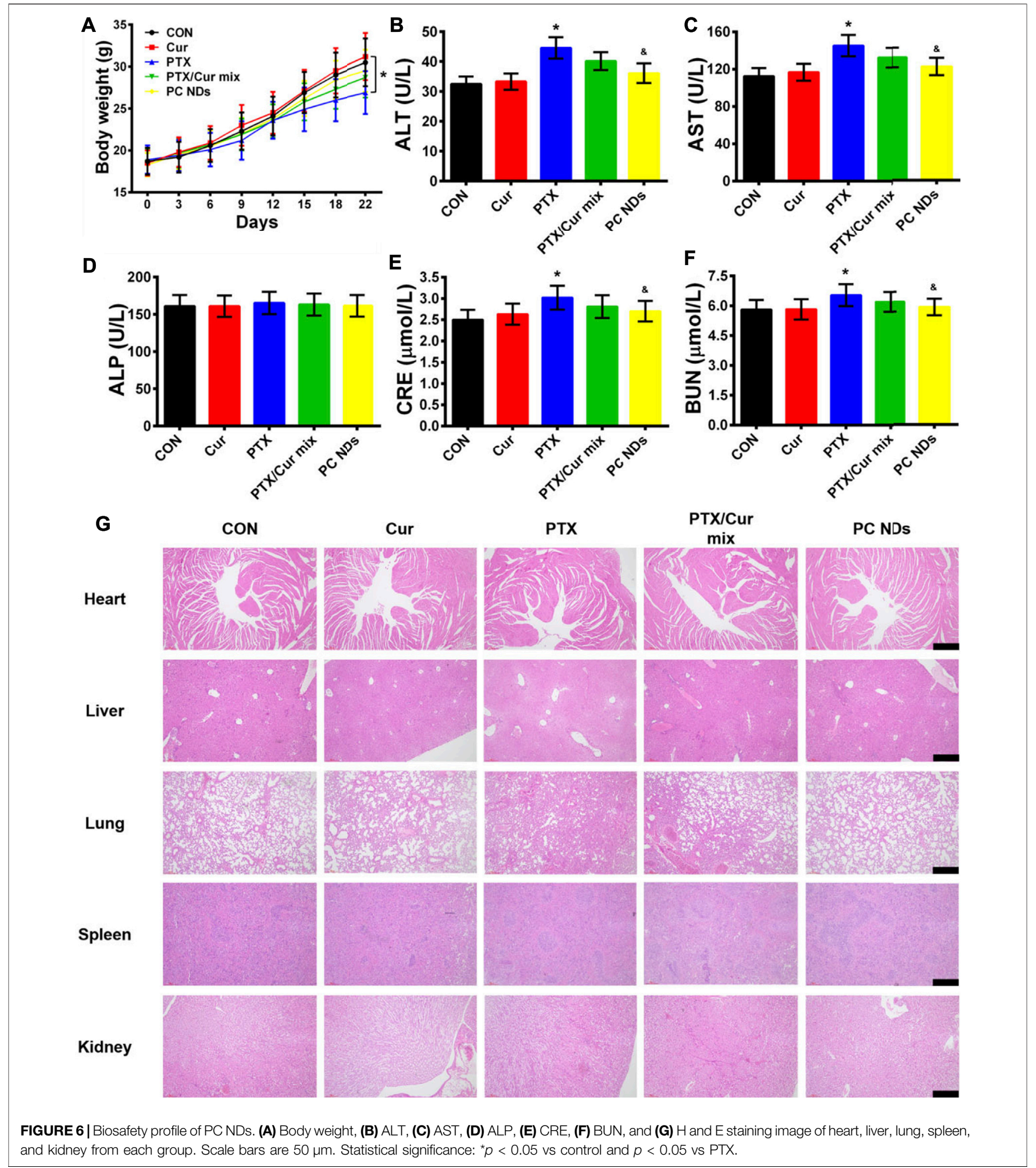

(Figures 5B-D), although free Cur did not significantly influence tumor growth. Furthermore, the PC NDs appear to provide substantially more promising results (vs the other formulations), based on a tumor growth inhibition rate of $80.36 \%$. Interestingly, free PTX provided considerable anti- tumor effects, while mice were suffered from a decrease in body weight and abnormal high levels of liver and kidney enzymes (ALT, AST, BUN, and CRE) (Figures 6A-F). In contrast, the PC ND group only exhibited a small decrease in body weight after 21 days of treatment and no histopathological 
changes in the liver, spleen, kidneys, heart, and lungs (Figures 6A-G) (Edwards et al., 2017; Farhood et al., 2019). Moreover, we observed severe lung metastasis in the PTX and PTX/Cur mix groups, which was not observed in the $\mathrm{PC}$ ND group (Supplementary Figure S5) (Sesarman et al., 2018; Tan and Norhaizan, 2019). Thus, co-delivery of PTX and Cur via the PC NDs might improve the efficacy of treatment for TNBC (vs free PTX alone), with less systemic toxicity observed in our mouse model, although the underlying mechanisms remain unclear.

In summary, we created biocompatible and carrier-free nanodrugs composed of PTX and Cur via a simple nanoprecipitation method. The PC NDs were preferentially taken up by TNBC cells and we also observed $\mathrm{pH}$-related drug release. The cytotoxicity assay revealed that the PC NDs had a greater effect on TNBC cells (vs free PTX), as well as less toxicity in normal cells. The in vivo data also clearly indicated that the PC NDs had considerably greater therapeutic efficacy than the free PTX/Cur mixture, with no signs of systemic toxicity. Therefore, the PC NDs might be a promising carrier-free strategy for safely and effectively delivering PTX and Cur to treat TNBC. Further studies are needed to determine whether this nanotherapeutic strategy holds clinical value.

\section{DATA AVAILABILITY STATEMENT}

The raw data supporting the conclusions of this article will be made available by the authors, without undue reservation, to any qualified researcher.

\section{REFERENCES}

Abu Samaan, T. M., Samec, M., Liskova, A., Kubatka, P., and Büsselberg, D. (2019). Paclitaxel's Mechanistic and Clinical Effects on Breast Cancer. Biomolecules 9 (12), 789. doi:10.3390/biom9120789

Akram, M., Iqbal, M., Daniyal, M., and Khan, A. U. (2017). Awareness and Current Knowledge of Breast Cancer. Biol. Res. 50 (1), 33. doi:10.1186/s40659-0170140-9

Baek, J.-S., and Cho, C.-W. (2017). A Multifunctional Lipid Nanoparticle for Codelivery of Paclitaxel and Curcumin for Targeted Delivery and Enhanced Cytotoxicity in Multidrug Resistant Breast Cancer Cells. Oncotarget 8 (18), 30369-30382. doi:10.18632/oncotarget.16153

Bergin, A. R. T., and Loi, S. (2019). Triple-negative Breast Cancer: Recent Treatment Advances. F1000Res 8, 1342. doi:10.12688/f1000research.18888.1

Bielopolski, D., Evron, E., Moreh-Rahav, O., Landes, M., Stemmer, S. M., and Salamon, F. (2017). Paclitaxel-induced Pneumonitis in Patients with Breast Cancer: Case Series and Review of the Literature. J. Chemother. 29 (2), 113-117. doi:10.1179/1973947815y.0000000029

Borges, G. A., Elias, S. T., Amorim, B., Lima, C. L., Coletta, R. D., Castilho, R. M., et al. (2020). Curcumin Downregulates the PI3K-AKT-mTOR Pathway and Inhibits Growth and Progression in Head and Neck Cancer Cells. Phytotherapy Res. 34 (12), 3311-3324. doi:10.1002/ptr.6780

Calaf, G., Ponce-Cusi, R., and Carrión, F. (2018). Curcumin and Paclitaxel Induce Cell Death in Breast Cancer Cell Lines. Oncol. Rep. 40 (4), 2381-2388. doi:10.3892/or.2018.6603

Chen, P., Huang, H.-P., Wang, Y., Jin, J., Long, W.-G., Chen, K., et al. (2019). Curcumin Overcome Primary Gefitinib Resistance in Non-small-cell Lung Cancer Cells through Inducing Autophagy-Related Cell Death. J. Exp. Clin. Cancer Res. 38 (1), 254. doi:10.1186/s13046-019-1234-8

Edwards, R. L., Luis, P. B., Varuzza, P. V., Joseph, A. I., Presley, S. H., Chaturvedi, R., et al. (2017). The Anti-inflammatory Activity of Curcumin Is Mediated by its

\section{ETHICS STATEMENT}

The animal study was reviewed and approved by Animal Care and Use Committee of Jilin University. Written informed consent was obtained from the owners for the participation of their animals in this study.

\section{AUTHOR CONTRIBUTIONS}

SZ, ZW, DS, and YZ designed the research. SZ, ZW, XA, JW, and $\mathrm{XZ}$ performed the research. SZ, XA, and JW analyzed the data. $\mathrm{SZ}$, DS, and YZ wrote the manuscript.

\section{FUNDING}

This study was supported by Scientific Development Program of Jilin Province (20180201054YY), Finance Department Program of Jilin Province (2020SCZT028), and the Fundamental Research Funds for the Central Universities.

\section{SUPPLEMENTARY MATERIAL}

The Supplementary Material for this article can be found online at: https://www.frontiersin.org/articles/10.3389/fbioe.2021.747637/ full\#supplementary-material

Oxidative Metabolites. J. Biol. Chem. 292 (52), 21243-21252. doi:10.1074/ jbc.ra117.000123

Fahad Ullah, M. (2019). Breast Cancer: Current Perspectives on the Disease Status. Adv. Exp. Med. Biol. 1152, 51-64. doi:10.1007/978-3-030-20301-6_4

Farhood, B., Mortezaee, K., Goradel, N. H., Khanlarkhani, N., Salehi, E., Nashtaei, M. S., et al. (2019). Curcumin as an Anti-inflammatory Agent: Implications to Radiotherapy and Chemotherapy. J. Cel. Physiol. 234 (5), 5728-5740. doi:10.1002/jcp. 27442

Ghasemi, F., Shafiee, M., Banikazemi, Z., Pourhanifeh, M. H., Khanbabaei, H., Shamshirian, A., et al. (2019). Curcumin Inhibits NF-kB and Wnt/ $\beta$-Catenin Pathways in Cervical Cancer Cells. Pathol. - Res. Pract. 215 (10), 152556. doi:10.1016/j.prp.2019.152556

Hamzehzadeh, L., Atkin, S. L., Majeed, M., Butler, A. E., and Sahebkar, A. (2018). The Versatile Role of Curcumin in Cancer Prevention and Treatment: A Focus on PI3K/ AKT Pathway. J. Cel. Physiol. 233 (10), 6530-6537. doi:10.1002/jcp.26620

Hiremath, C. G., Heggnnavar, G. B., Kariduraganavar, M. Y., and Hiremath, M. B. (2019). Co-delivery of Paclitaxel and Curcumin to Foliate Positive Cancer Cells Using Pluronic-Coated Iron Oxide Nanoparticles. Prog. Biomater. 8 (3), 155-168. doi:10.1007/s40204-019-0118-5

Hu, H., Yang, C., Zhang, F., Li, M., Tu, Z., Mu, L., et al. (2021). A Versatile and Robust Platform for the Scalable Manufacture of Biomimetic Nanovaccines. Adv. Sci. 8, 2002020. doi:10.1002/advs.202002020

Hupfer, M. L., Herrmann-Westendorf, F., Dietzek, B., and Presselt, M. (2021). In Situ Photothermal Deflection Spectroscopy Revealing Intermolecular Interactions upon Self-Assembly of Dye Monolayers. Analyst 146 (16), 5033-5036. doi:10.1039/d1an00582k

Kang, C., and Syed, Y. Y. (2020). Atezolizumab (In Combination with NabPaclitaxel): A Review in Advanced Triple-Negative Breast Cancer. Drugs 80 (6), 601-607. doi:10.1007/s40265-020-01295-y

Keyvani-Ghamsari, S., Khorsandi, K., and Gul, A. (2020). Curcumin Effect on Cancer Cells' Multidrug Resistance: An Update. Phytotherapy Res. 34 (10), 2534-2556. doi:10.1002/ptr.6703 
Kumar, P., and Aggarwal, R. (2016). An Overview of Triple-Negative Breast Cancer. Arch. Gynecol. Obstet. 293 (2), 247-269. doi:10.1007/s00404-0153859-y

Li, M., Sun, X., Zhang, N., Wang, W., Yang, Y., Jia, H., et al. (2018). NIR-activated Polydopamine-Coated Carrier-free "Nanobomb" for In Situ On-Demand Drug Release. Adv. Sci. 5 (7), 1800155. doi:10.1002/advs.201800155

Li, Q., Wen, J., Liu, C., Jia, Y., Wu, Y., Shan, Y., et al. (2019). Graphenenanoparticle-based Self-Healing Hydrogel in Preventing Postoperative Recurrence of Breast Cancer. ACS Biomater. Sci. Eng. 5 (2), 768-779. doi:10.1021/acsbiomaterials.8b01475

Liao, J., Han, R., Wu, Y., and Qian, Z. (2021). Review of a New Bone Tumor Therapy Strategy Based on Bifunctional Biomaterials. Bone Res. 9 (1), 1-13. doi:10.1038/s41413-021-00139-z

Lyons, T. G. (2019). Targeted Therapies for Triple-Negative Breast Cancer. Curr. Treat. Options. Oncol. 20 (11), 82. doi:10.1007/s11864-019-0682-x

Maiti, P., Plemmons, A., and Dunbar, G. L. (2019). Combination Treatment of Berberine and Solid Lipid Curcumin Particles Increased Cell Death and Inhibited PI3K/Akt/mTOR Pathway of Human Cultured Glioblastoma Cells More Effectively Than Did Individual Treatments. PloS one 14 (12), e0225660. doi:10.1371/journal.pone.0225660

McDonald, E. S., Clark, A. S., Tchou, J., Zhang, P., and Freedman, G. M. (2016). Clinical Diagnosis and Management of Breast Cancer. J. Nucl. Med. 57 (Suppl. 1), 9s-16s. doi:10.2967/jnumed.115.157834

Mittendorf, E. A., Zhang, H., Barrios, C. H., Saji, S., Jung, K. H., Hegg, R., et al. (2020). Neoadjuvant Atezolizumab in Combination with Sequential NabPaclitaxel and Anthracycline-Based Chemotherapy versus Placebo and Chemotherapy in Patients with Early-Stage Triple-Negative Breast Cancer (IMpassion031): a Randomised, Double-Blind, Phase 3 Trial. The Lancet 396 (10257), 1090-1100. doi:10.1016/s0140-6736(20)31953-x

Saghatelyan, T., Tananyan, A., Janoyan, N., Tadevosyan, A., Petrosyan, H., Hovhannisyan, A., et al. (2020). Efficacy and Safety of Curcumin in Combination with Paclitaxel in Patients with Advanced, Metastatic Breast Cancer: A Comparative, Randomized, Double-Blind, Placebo-Controlled Clinical Trial. Phytomedicine 70, 153218. doi:10.1016/j.phymed.2020.153218

Saha, S., Adhikary, A., Bhattacharyya, P., Das, T., and Sa, G. (2012). Death by Design: where Curcumin Sensitizes Drug-Resistant Tumours. Anticancer Res. 32 (7), 2567-2584.

Schmid, P., Adams, S., Rugo, H. S., Schneeweiss, A., Barrios, C. H., Iwata, H., et al. (2018). Atezolizumab and Nab-Paclitaxel in Advanced Triple-Negative Breast Cancer. N. Engl. J. Med. 379 (22), 2108-2121. doi:10.1056/NEJMoa1809615

Sesarman, A., Tefas, L., Sylvester, B., Licarete, E., Rauca, V., Luput, L., et al. (2018). Anti-angiogenic and Anti-inflammatory Effects of Long-Circulating Liposomes Co-encapsulating Curcumin and Doxorubicin on C26 Murine colon Cancer Cells. Pharmacol. Rep. 70 (2), 331-339. doi:10.1016/j.pharep.2017.10.004

Shao, D., Zhang, F., Chen, F., Zheng, X., Hu, H., Yang, C., et al. (2020). Biomimetic Diselenide-Bridged Mesoporous Organosilica Nanoparticles as an X-rayResponsive Biodegradable Carrier for Chemo-Immunotherapy. Adv. Mater. 32 (50), 2004385. doi:10.1002/adma.202004385

Subramaniam, D., Kaushik, G., Dandawate, P., and Anant, S. (2018). Targeting Cancer Stem Cells for Chemoprevention of Pancreatic Cancer. Cmc 25 (22), 2585-2594. doi:10.2174/0929867324666170127095832

Sun, M., Zhang, Y., He, Y., Xiong, M., Huang, H., Pei, S., et al. (2019). Green Synthesis of Carrier-free Curcumin Nanodrugs for Light-Activated Breast Cancer Photodynamic Therapy. Colloids Surf. B: Biointerfaces 180, 313-318. doi:10.1016/j.colsurfb.2019.04.061

Tan, B. L., and Norhaizan, M. E. (2019). Curcumin Combination Chemotherapy: the Implication and Efficacy in Cancer. Molecules 24 (14), 2527. doi:10.3390/ molecules 24142527
Xiong, K., Zhang, Y., Wen, Q., Luo, J., Lu, Y., Wu, Z., et al. (2020). Co-delivery of Paclitaxel and Curcumin by Biodegradable Polymeric Nanoparticles for Breast Cancer Chemotherapy. Int. J. pharmaceutics 589, 119875. doi:10.1016/ j.ijpharm.2020.119875

Yang, M.-Y., Zhao, R.-R., Fang, Y.-F., Jiang, J.-L., Yuan, X.-T., and Shao, J.-W. (2019). Carrier-free Nanodrug: A Novel Strategy of Cancer Diagnosis and Synergistic Therapy. Int. J. pharmaceutics 570, 118663. doi:10.1016/ j.ijpharm.2019.118663

Yeo, S. K., and Guan, J.-L. (2017). Breast Cancer: Multiple Subtypes within a Tumor? Trends Cancer 3 (11), 753-760. doi:10.1016/j.trecan.2017.09.001

Yin, L., Duan, J.-J., Bian, X.-W., and Yu, S.-c. (2020). Triple-negative Breast Cancer Molecular Subtyping and Treatment Progress. Breast Cancer Res. 22 (1), 61. doi:10.1186/s13058-020-01296-5

Yoshida, K., Toden, S., Ravindranathan, P., Han, H., and Goel, A. (2017). Curcumin Sensitizes Pancreatic Cancer Cells to Gemcitabine by Attenuating PRC2 Subunit EZH2, and the lncRNA PVT1 Expression. Carcinogenesis 38 (10), 1036-1046. doi:10.1093/carcin/bgx065

Zhang, F., Chen, F., Yang, C., Wang, L., Hu, H., Li, X., et al. (2021). Coordination and Redox Dual-Responsive Mesoporous Organosilica Nanoparticles Amplify Immunogenic Cell Death for Cancer Chemoimmunotherapy. Small 17, 2100006. doi:10.1002/smll.202100006

Zhang, F., Zhang, M., Zheng, X., Tao, S., Zhang, Z., Sun, M., et al. (2018). Berberine-based Carbon Dots for Selective and Safe Cancer Theranostics. RSC Adv. 8 (3), 1168-1173. doi:10.1039/c7ra12069a

Zhao, M.-D., Li, J.-Q., Chen, F.-Y., Dong, W., Wen, L.-J., Fei, W.-D., et al. (2019). Co-Delivery of Curcumin and Paclitaxel by "Core-Shell" Targeting Amphiphilic Copolymer to Reverse Resistance in the Treatment of Ovarian Cancer. Ijn Vol. 14, 9453-9467. doi:10.2147/ijn.s224579

Zheng, X., Zhang, F., Shao, D., Zhang, Z., Cui, L., Zhang, J., et al. (2018). Gramscale Production of Carrier-free Fluorescent Berberine Microrods for Selective Liver Cancer Therapy. BioFactors 44 (5), 496-502. doi:10.1002/ biof. 1450

Zhou, M., Wei, W., Chen, X., Xu, X., Zhang, X., and Zhang, X. (2019). pH and Redox Dual Responsive Carrier-free Anticancer Drug Nanoparticles for Targeted Delivery and Synergistic Therapy. Nanomedicine: Nanotechnology, Biol. Med. 20, 102008. doi:10.1016/j.nano.2019.04.011

Zusso, M., Lunardi, V., Franceschini, D., Pagetta, A., Lo, R., Stifani, S., et al. (2019). Ciprofloxacin and Levofloxacin Attenuate Microglia Inflammatory Response via TLR4/NF-kB Pathway. J. Neuroinflammation 16 (1), 148. doi:10.1186/ s12974-019-1538-9

Conflict of Interest: The authors declare that the research was conducted in the absence of any commercial or financial relationships that could be construed as a potential conflict of interest.

Publisher's Note: All claims expressed in this article are solely those of the authors and do not necessarily represent those of their affiliated organizations, or those of the publisher, the editors and the reviewers. Any product that may be evaluated in this article, or claim that may be made by its manufacturer, is not guaranteed or endorsed by the publisher.

Copyright $\odot 2021$ Zuo, Wang, An, Wang, Zheng, Shao and Zhang. This is an openaccess article distributed under the terms of the Creative Commons Attribution License (CC BY). The use, distribution or reproduction in other forums is permitted, provided the original author(s) and the copyright owner(s) are credited and that the original publication in this journal is cited, in accordance with accepted academic practice. No use, distribution or reproduction is permitted which does not comply with these terms. 